\title{
Ranking Programs using Black Box Testing
}

\author{
Koen Claessen \\ Chalmers University of \\ Technology \\ Gothenburg, Sweden. \\ koen@chalmers.se
}

\author{
John Hughes \\ Chalmers University of \\ Technology and Quviq AB \\ Gothenburg, Sweden. \\ rjmh@chalmers.se
}

\author{
Michał Pałka \\ Chalmers University of \\ Technology \\ Gothenburg, Sweden. \\ michal.palka@chalmers.se
}

\author{
Nick Smallbone \\ Chalmers University of \\ Technology \\ Gothenburg, Sweden. \\ nicsma@chalmers.se
}

\author{
Hans Svensson \\ Chalmers University of \\ Technology and Quviq AB \\ Gothenburg, Sweden. \\ hanssv@chalmers.se
}

\begin{abstract}
We present an unbiased method for measuring the relative quality of different solutions to a programming problem. Our method is based on identifying possible bugs from program behaviour through black-box testing. The main motivation for such a method is its use in experimental evaluation of software development methods. We report on the use of our method in a small-scale such experiment, which was aimed at evaluating the effectiveness of property-based testing vs. unit testing in software development.
\end{abstract}

\section{Categories and Subject Descriptors}

D.2.8 [Software Engineering]: Metrics

\section{General Terms}

Measurement

\section{INTRODUCTION}

Property-based testing is an approach to testing software against a formal specification, consisting of universally quantified properties which supply both test data generators and test oracles. QuickCheck is a property-based testing tool first developed for Haskell [6], and which forms the basis for a commercial tool developed by Quviq [1]. As a simple example, using QuickCheck, a programmer could specify that list reversal is its own inverse like this,

prop_reverse (xs : : [Integer]) =

reverse $($ reverse xs) $==x s$

which defines a property called prop_reverse which is universally quantified over all lists of integers xs. Given such a property, QuickCheck generates random values for xs as test data, and uses the body of the property as an oracle

Permission to make digital or hard copies of all or part of this work for personal or classroom use is granted without fee provided that copies are not made or distributed for profit or commercial advantage and that copies bear this notice and the full citation on the first page. To copy otherwise, to republish, to post on servers or to redistribute to lists, requires prior specific permission and/or a fee.

AST 2010 May 2-8 2010, Cape Town, South Africa

Copyright 2010 ACM 978-1-60558-970-1/10/05 ...\$10.00. to decide whether each test has passed. When a test fails, QuickCheck shrinks the failing test case, searching systematically for a minimal failing example, in a way similar to delta-debugging [17]. The resulting minimal failing case usually makes diagnosing a fault easy. For example, if the programmer erroneously wrote

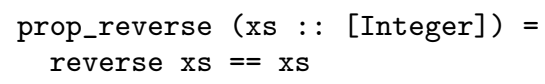

then QuickCheck would report the minimal counterexample $[0,1]$, since at least two different elements are needed to violate the property, and the two smallest different integers are 0 and 1.

The idea of testing code against general properties, rather than specific test cases, is an appealing one which also underlies Tillmann and Schulte's parameterized unit tests [15] and the Pex tool [14] (although the test case generation works differently). We believe firmly that it brings a multitude of benefits to the developer, improving quality and speeding development by revealing problems faster and earlier. Yet claims such as this are easy to make, but hard to prove. And it is not obvious that property-based testing must be superior to traditional test automation. Among the possible disadvantages of QuickCheck testing are:

- it is often necessary to write test data generators for problem-specific data structures - code which is not needed at all in traditional testing.

- the developer must formulate a formal specification, which is conceptually more difficult than just predicting the correct output in specific examples.

- randomly generated test cases might potentially be less effective at revealing errors than carefully chosen ones.

Thus an empirical comparison of property-based testing against other methods is warranted.

Our overall goal is to evaluate property-based testing as a development tool, by comparing programs developed by students using QuickCheck for testing, against programs developed for the same problem using HUnit [8] - a unit testing framework for Haskell similar to the popular JUnit tool for Java programmers [9]. We have not reached this goal yet - we have carried out a small-scale experiment, but we 
need more participants to draw statistically significant conclusions. However, we have identified an important problem to solve along the way: how should we rank student solutions against each other, without introducing experimental bias?

Our intention is to rank solutions by testing them: those that pass the most tests will be ranked the highest. But the choice of test suite is critical. It is tempting to use QuickCheck to test student solutions against our own properties, using the proportion of tests passed as a measure of quality - but this would risk experimental bias in two different ways:

- By using one of the tools in the comparison to grade solutions, we might unfairly bias the experiment to favour that tool,

- The ranking of solutions could depend critically on the distribution of random tests, which is rather arbitrary.

Unfortunately, a manually constructed set of test cases could also introduce experimental bias. If we were to include many similar tests of a particular kind, for example, then handling that kind of test successfully would carry more weight in our assessment of solutions than handling other kinds of test.

Our goal in this paper, thus, is to develop a way of ranking student solutions by testing that leaves no room for the experimenter's bias to affect the result. We will do so by generating a set of test cases from the submissions themselves, based on a simple "bug model" presented in section 3, such that each test case tests for one bug. We then rank solutions by the number of bugs they contain. QuickCheck is used to help find this set of test cases, but in such a way that the distribution of random tests is of almost no importance.

Contribution The main contribution of this paper is the ranking method we developed. As evidence that the ranking is reasonable, we also present the results of our small-scale experiment, in which solutions to three different problems are compared in this way.

The remainder of the paper is structured as follows. In the next section we briefly describe the experiment we carried out. In section 3 we explain and motivate our ranking method. Section 4 analyses the results obtained. In section 5 we discuss related work, and we conclude in section 6 .

\section{THE EXPERIMENT}

We designed an experiment to test the hypothesis that "Property-based testing is more effective than unit testing, as a tool during software development", using QuickCheck as the property-based testing tool, and HUnit as the unit testing tool. We used a replicated project study[3], where in a controlled experiment a group of student participants individually solved three different programming tasks. We planned the experiment in accordance to best practice for such experiments; trying not to exclude participants, assigning the participants randomly to tools, using a variety of programming tasks, and trying our best not to influence the outcome unnecessarily. We are only evaluating the final product, thus we are not interested in process aspects in this study.

In the rest of this section we describe in more detail how we planned and executed the experiment, we also motivate the choice of programming assignments given to the participants.

\subsection{Experiment overview}

We planned an experiment to be conducted during one day. Since we expected participants to be unfamiliar with at least one of the tools in the comparison, we devoted the morning to a training session in which the tools were introduced to the participants. The main issue in the design of the experiment was the programming task (or tasks) to be given to the participants. Using several different tasks would yield more data points, while using one single (bigger) task would give us data points of higher quality. We decided to give three separate tasks to the participants, mostly because by doing this, and selecting three different types of problems, we could reduce the risk of choosing a task particularly suited to one tool or the other. All tasks were rather small, and require only 20-50 lines of Haskell code to implement correctly.

To maximize the number of data points we decided to assign the tasks to individuals instead of forming groups. Repeating the experiments as a pair-programming assignment would also be interesting.

\subsection{Programming assignments}

We constructed three programming assignments. We tried to choose problems from three separate categories; one datastructure implementation problem, one search/algorithmic problem, and one slightly tedious string manipulation task.

Problem 1: E-mail anonymizer In this task the participants were asked to write a sanitizing function anonymize which blanks out E-mail addresses in a string. For example,

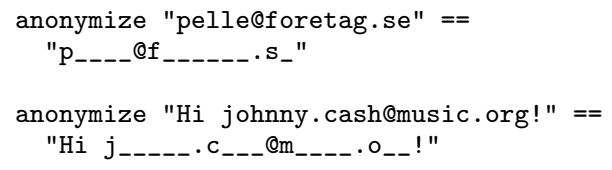

The function should identify all e-mail addresses in the input, change them, but leave all other text untouched. This is a simple problem, but with a lot of tedious cases.

Problem 2: Interval sets In this task the participants were asked to implement a compact representation of sets of integers based on lists of intervals, represented by the type IntervalSet $=[($ Int, Int $)]$, where for example the set $\{1,2,3,7,8,9,10\}$ would be represented by the list $[(1,3),(7,10)]$. The participants were instructed to implement a family 7 of functions for this data type (empty, member, insert, delete, merge). There are many special cases to consider-for example, inserting an element between two intervals may cause them to merge into one.

Problem 3: Cryptarithm In this task the students were asked to write a program that solves puzzles like this one:

SEND
MORE
----+
MONEY

The task is to assign a mapping from letters to (unique) digits, such that the calculation makes sense. (In the example $\mathrm{M}=1,0=0, \mathrm{~S}=9, \mathrm{R}=8, \mathrm{E}=5, \mathrm{~N}=6, \mathrm{Y}=$ $2, \mathrm{D}=7$ ). Solving the puzzle is complicated by the fact that there might be more than one solution and that there are problems for which there is no solution. This is a search problem, which requires an algorithm with some level of sophistication to be computationally feasible. 


\subsection{The participants}

Since the university (Chalmers University of Technology, Gothenburg, Sweden) teaches Haskell, this was the language we used in the experiment. We tried to recruit students with (at least) a fair understanding of functional programming. This we did because we believed that too inexperienced programmers would not be able to benefit from either QuickCheck or HUnit. The participants were recruited by advertising on campus, email-messages sent to students from the previous Haskell-course and announcements in different ongoing courses. Unfortunately the only available date collided with exams at the university, which lowered the number of potential participants. In the end we got only 13 participants. This is too few to draw statistically significant conclusions, but on the other hand it is a rather manageable number of submissions to analyze in a greater detail. Most of the participants were at a level where they had passed (often with honor) a 10-week programming course in Haskell.

\subsection{Assigning the participants into groups}

We assigned the participants randomly (by lot) into two groups, one group using QuickCheck and one group using HUnit.

\subsection{Training the participants}

The experiment started with a training session for the participants. The training was divided into two parts, one joint session, and one session for the specific tool. In the first session, we explained the purpose and the underlying hypothesis for the experiment. We also clearly explained that we were interested in software quality rather than development time. The participants were encouraged to use all of the allocated time to produce the best software possible.

In the second session the groups were introduced to their respective testing tools, by a lecture and practical session. Both sessions lasted around 60 minutes.

\subsection{Programming environment}

Finally, with everything set up, the participants were given the three different tasks with a time limit of 50 minutes for each of the tasks. The participants were each given a computer equipped with GHC (the Haskell compiler) [13], both the testing tools, and documentation. The computers were connected to the Internet, but since the participants were aware of the purpose of the study and encouraged not to use other tools than the assigned testing tool it is our belief this did not affect the outcome of the experiment. ${ }^{1}$

\subsection{Data collection and reduction}

From the experiments we collected the implementations as well as the testing code written by each participant.

Manual grading of implementations Each of the three tasks were graded by an experienced Haskell programmer. We graded each implementation on a scale 0-10, just as we would have graded an exam-question. Since the tasks were reasonably small, and the number of participants manageable, this was feasible. To prevent any possible bias, the grader was not allowed to see the testing code and thus he

\footnotetext{
${ }^{1}$ Why not simply disconnect the computers from the Internet? Because we used an on-line submission system, as well as documentation and software from network file systems.
}

could not know whether each student was using QuickCheck or HUnit.

Automatic ranking The implementations of each problem were subjected to an analysis that we present in section 3 .

We had several students submit uncompileable code. ${ }^{2}$ In those cases, we made the code compile by for example removing any ill-formed program fragments. This was because such a program might be partly-working, and deserve a reasonable score; we thought it would be unfair if it got a score of zero simply because it (say) had a syntax error.

Grading of test suites We also graded participants' testing code. Each submission was graded by hand by judging the completeness of the test suite-and penalised for missing cases (for HUnit) or incomplete specifications (for QuickCheck). As we did not instruct the students to use TDD, there was no penalty for not testing a function if that function was not implemented.

Cross-comparison of tests We naturally wanted to automatically grade students' test code too - not least, because a human grader may be biased towards QuickCheck or HUnit tests. Our approach was simply to take each student's test suite, and run it against all of the submissions we had; for every submission the test suite found a bug in, it scored one point.

We applied this method successfully to the interval sets problem. However, for the anonymizer and cryptarithm problems, many students performed white box testing, testing functions that were internal to their implementation; therefore we were not able to transfer test suites from one implementation to another, and we had to abandon the idea for these problems.

\section{EVALUATION METHOD}

We assume we have a number of student answers to evaluate, $A_{1} \ldots A_{n}$, and a perfect solution $A_{0}$, each answer being a program mapping a test case to output. We assume that we have a test oracle which can determine whether or not the output produced by an answer is correct, for any possible test case. Such an oracle can be expressed as a QuickCheck property - if the correct output is unique, then it is enough to compare with $A_{0}$ 's output, otherwise something more complex is required. Raising an exception, or falling into a loop ${ }^{3}$, is never correct behaviour. We can thus determine, for an arbitrary test case, which of the student answers pass the test.

We recall that the purpose of our automatic evaluation method is to find a set of test cases that is as unbiased as possible. In particular, we want to avoid counting multiple test cases that are equivalent, in the sense that they trigger the same bug.

Thus, we aim to "count the bugs" in each answer, using black-box testing alone. How, then, should we define a "bug"? We cannot refer to errors at specific places in the source code, since we use black-box testing only-we must define

\footnotetext{
${ }^{2}$ Since we asked students to submit their code at a fixed time, some students submitted in the middle of making changes.

${ }^{3}$ detecting a looping program is approximated by an appropriately chosen timeout
} 
a "bug" in terms of the program behaviour. We take the following as our bug model:

- A bug causes a program to fail for a set of test cases. Given a bug $b$, we write the set of test cases that it causes to fail as BugTests(b). (Note that it is possible that the same bug $b$ occurs in several different programs.)

- A program $p$ will contain a set of bugs, Bugs $(p)$. The set of test cases that $p$ fails for will be

$$
\text { FailingTests }(p)=\quad \bigcup_{b \in \operatorname{Bugs}(p)} \operatorname{BugTests}(b)
$$

It is quite possible, of course, that two different errors in the source code might manifest themselves in the same way, causing the same set of tests to fail. We will treat these as the same bug, quite simply because there is no way to distinguish them using black-box testing.

It is also possible that two different bugs in combination might "cancel each other out" in some test cases, leading a program containing both bugs to behave correctly, despite their presence. We cannot take this possibility into account, once again because black-box testing cannot distinguish correct output produced "by accident" from correct output produced correctly. We believe the phenomenon, though familiar to developers, is rare enough not to influence our results strongly.

Our approach is to analyze the failures of the student answers, and use them to infer the existence of possible bugs $B u g s$, and their failure sets. Then we shall rank each answer program $A_{i}$ by the number of these bugs that the answer appears to contain:

$$
\operatorname{rank}\left(A_{i}\right)=\mid\left\{b \in \text { Bugs } \mid \text { BugTests }(b) \subseteq \text { FailingTests }\left(A_{i}\right)\right\} \mid
$$

In general, there are many ways of explaining program failures via a set of bugs. The most trivial is to take each answer's failure set Failing Tests $\left(A_{i}\right)$ to represent a different possible bug; then the rank of each answer would be the number of other (different) answers that fail on a strictly smaller set of inputs. However, we reject this idea as too crude, because it gives no insight into the nature of the bugs present. We shall aim instead to find a more refined set of possible bugs, in which each bug explains a small set of "similar" failures.

Now, let us define the failures of a test case to be the set of answers that it provokes to fail:

$$
\text { AnswersFailing }(t)=\left\{A_{i} \mid t \in \text { FailingTests }\left(A_{i}\right)\right\}
$$

We insist that if two test cases $t_{1}$ and $t_{2}$ provoke the same answers to fail, then they are equivalent with respect to the bugs we infer:

$$
\begin{aligned}
& \text { AnswersFailing }\left(t_{1}\right)=\text { AnswersFailing }\left(t_{2}\right) \Longrightarrow \\
& \quad \forall b \in \text { Bugs. } t_{1} \in \text { BugTests }(b) \Leftrightarrow t_{2} \in \operatorname{BugTests}(b)
\end{aligned}
$$

We will not distinguish such a pair of test cases, because there is no evidence from the answers that could justify doing so. Thus we can partition the space of test cases into subsets that behave equivalently with respect to our answers. By identifying bugs with these partitions (except, if it exists, the partition which causes no answers to fail), then we obtain a maximal set of bugs that can explain the failures we observe. No other set of bugs can be more refined than this without distinguishing inputs that should not be distinguished.
However, we regard this partition as a little too refined. Consider two answers $A_{1}$ and $A_{2}$, and three partitions $B$, $B_{1}$ and $B_{2}$, such that

$$
\begin{aligned}
& \forall t \in B . \text { AnswersFailing }(t)=\left\{A_{1}, A_{2}\right\} \\
& \forall t \in B_{1} . \text { AnswersFailing }(t)=\left\{A_{1}\right\} \\
& \forall t \in B_{2} . \text { AnswersFailing }(t)=\left\{A_{2}\right\}
\end{aligned}
$$

Clearly, one possibility is that there are three separate bugs represented here, and that

$$
\begin{aligned}
& \operatorname{Bugs}\left(A_{1}\right)=\left\{B, B_{1}\right\} \\
& \operatorname{Bugs}\left(A_{2}\right)=\left\{B, B_{2}\right\}
\end{aligned}
$$

But another possibility is that there are only two different bugs represented, $B_{1}^{\prime}=B \cup B_{1}$ and $B_{2}^{\prime}=B \cup B_{2}$, and that each $A_{i}$ just has one bug, $B_{i}^{\prime}$. In this case, test cases in $B$ can provoke either bug. Since test cases which can provoke several different bugs are quite familiar, then we regard the latter possibility as more plausible than the former. We choose therefore to ignore any partitions whose failing answers are the union of those of a set of other partitions; we call these partitions redundant, and we consider it likely that the test cases they contain simply provoke several bugs at once. In terms of our bug model, we combine such partitions with those representing the individual bugs whose union explains their failures. Note, however, that if a third answer $A_{3}$ only fails for inputs in $B$, then we consider this evidence that $B$ does indeed represent an independent bug (since $\left\{A_{1}, A_{2}, A_{3}\right\}$ is not the union of $\left\{A_{1}\right\}$ and $\left\{A_{2}\right\}$ ), and that answers $A_{1}$ and $A_{2}$ therefore contain two bugs each.

Now, to rank our answers we construct a test suite containing one test case from each of the remaining partitions, count the tests that each answer fails, and assign ranks accordingly.

In practice, we find the partitions by running a very large number of random tests. We maintain a set of test cases Suite, each in a different partition. For each newly generated test case $t$, we test all of the answers to compute AnswersFailing $(t)$. We then test whether the testcase is redundant in the sense described above:

$$
\begin{aligned}
& \begin{array}{l}
\text { Redundant }(t, \text { Suite }) \hat{=} \\
\text { AnswersFailing }(t)=
\end{array} \\
& \qquad\left\{\begin{array}{l|l}
\text { AnswersFailing }\left(t^{\prime}\right) & \begin{array}{l}
t^{\prime} \in \text { Suite }, \\
\text { AnswersFailing }\left(t^{\prime}\right) \subseteq \\
\text { AnswersFailing }(t)
\end{array}
\end{array}\right\}
\end{aligned}
$$

Whenever $t$ is not redundant, i.e. when Redundant $(t$, Suite) evaluates to False, then we apply QuickCheck's shrinking to find a minimal $t_{\text {min }}$ that is not redundant with respect to Suite - which is always possible, since if we cannot find any smaller test case which is irredundant, then we can just take $t$ itself. Then we add $t_{\min }$ to Suite, and remove any $t^{\prime} \in$ Suite such that Redundant $\left(t^{\prime},\left(\right.\right.$ Suite $\left.\left.-t^{\prime}\right) \cup\left\{t_{\text {min }}\right\}\right)$. (Shrinking at this point probably helps us to find test cases that provoke a single bug rather than several - "probably" since a smaller test case is likely to provoke fewer bugs than a larger one, but of course there is no guarantee of this).

We continue this process until a large number of random tests fail to add any test cases to Suite. At this point, we assume that we have found one test case for each irredundant input partition, and we can use our test suite to rank answers.

Note that this method takes no account of the sizes of the partitions involved - we count a bug as a bug, whether 
it causes a failure for only one input value, or for infinitely many. Of course, the severity of bugs in practice may vary dramatically depending on precisely which inputs they cause failures for-but taking this into account would make our results dependent on value judgements about the importance of different kinds of input, and these value judgements would inevitably introduce experimental bias.

In the following section, we will see how this method performs in practice.

\section{ANALYSIS}

We adopted the statistical null hypothesis to be that there is no difference in quality between programs developed using QuickCheck and programs developed using HUnit. The aim of our analysis will be to establish whether the samples we got are different in a way which cannot be explained by coincidence.

We collected solutions to all three tasks programmed by 13 students, 7 of which were assigned to the group using QuickCheck and the remaining 6 to one using HUnit. In this section we will refer to the answers (solutions to tasks) as $A 1$ to $A 13$. Since the submissions have been anonymized, numbering of answers have also been altered and answers $A 1$ to different problems correspond to submissions of different participants. For each task there is also a special answer $A 0$ which is the model answer which we use as the testing oracle. For the anonymizer, we also added the identity function for comparison as $A 14$, and for the interval sets problem we added a completely undefined solution as $A 14$.

\subsection{Automatic Ranking of Solutions}

We ranked all solutions according to the method outlined in section 3. The ranking method produced a test-suite for each of the three tasks and assigned the number of failing tests to each answer of every task. The final score that we used for evaluation of answers was the number of successful runs on tests from the test-suite. The generated test suites are shown in Figure 1. Every test in the test suite causes some answer to fail; for example delete 0 [] is the simplest test that causes answers that did not implement the delete function to fail. These test cases have been shrunk by QuickCheck, which is why the only letter to appear in the anonymizer test cases is 'a', and why the strings are so short ${ }^{4}$.

Figures 2 to 4 visualize the test results. Each node represents a set of answers which pass precisely the same tests. An arrow from one node to another means that the answers at the target of the arrow pass a subset of the tests that the answers at the source of the arrow pass. Arrows are labelled with a test case that distinguishes the source and target, and the number of other such test cases in brackets. For instance, we can read from Figure 2 that $A 2$ fails three more tests than $A 7$, and that it fails on the input string "@" whereas $A 7$ succeeds on it. Thus these figures visualize a "correctness partial order" on the submitted answers.

The top node of each graph represents the entirely correct solutions, including the model answer A0. The bottom node represents incomplete solutions, in which the main functions

\footnotetext{
${ }^{4}$ Because Haskell encourages the use of dynamic datastructures, then none of the solutions could encounter a buffer overflow or other error caused by fixed size arrays. As a result, there is no need for tests with very long strings.
}

\begin{tabular}{|c|c|c|}
\hline Anon & IntSet & Crypt \\
\hline "1" & $\begin{array}{lll}\text { member } & 0 & {[}\end{array}$ & $\mathrm{b}+\mathrm{b}=\mathrm{c}$ \\
\hline$" \backslash \mathrm{n} "$ & member $0[(-2,2)]$ & $a+a=a$ \\
\hline "@" & member $2[(1,1)]$ & $a+b=a b$ \\
\hline "a" & member $0[(-3,-3),(0,4)]$ & $a a+a=b a c$ \\
\hline "\&@" & insert $0\left[\begin{array}{l}0 \\
0\end{array}\right.$ & \\
\hline ".@" & insert $-1[(1,1)]$ & \\
\hline "@@" & insert $0[(-2,0)]$ & \\
\hline ".৫৫" & insert $1[(-2,0)]$ & \\
\hline "@_a" & insert $2[(0,0)]$ & \\
\hline$" @ a="$ & delete 0[] & \\
\hline "_\&" & delete $0[(0,0)]$ & \\
\hline "a@a" & delete $0[(0,1)]$ & \\
\hline "\#@\&@" & merge []$\quad[]$ & \\
\hline ".a@\#" & merge []$[(-1,0)]$ & \\
\hline "a@_a" & merge $[(0,0)] \quad[(0,0)]$ & \\
\hline "a@aa" & merge $[(-1,0),(2,3)][(-1,0)]$ & \\
\hline
\end{tabular}

Figure 1: Generated test suites.

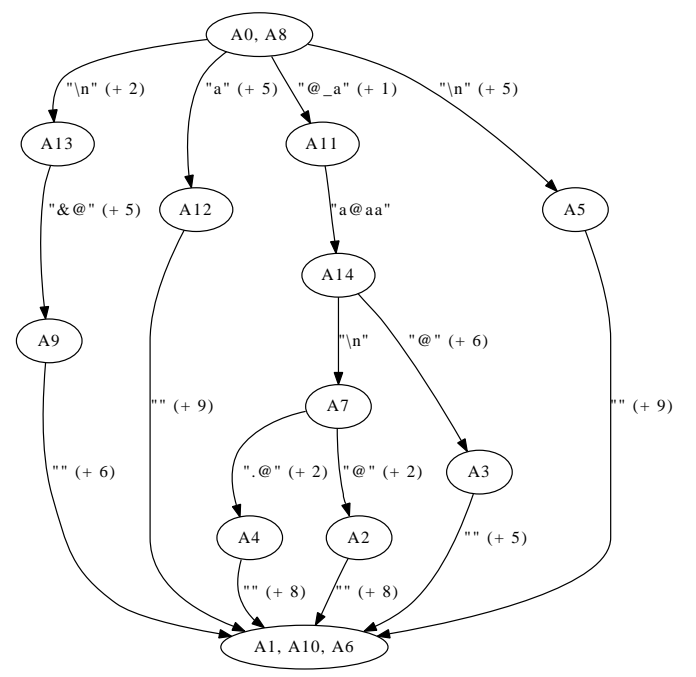

Figure 2: Relative correctness of anonymizer answers.

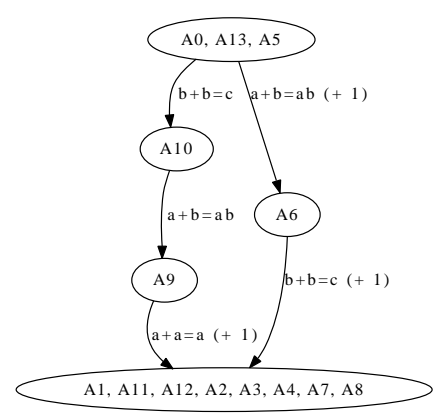

Figure 4: Relative correctness of cryptarithm answers. 


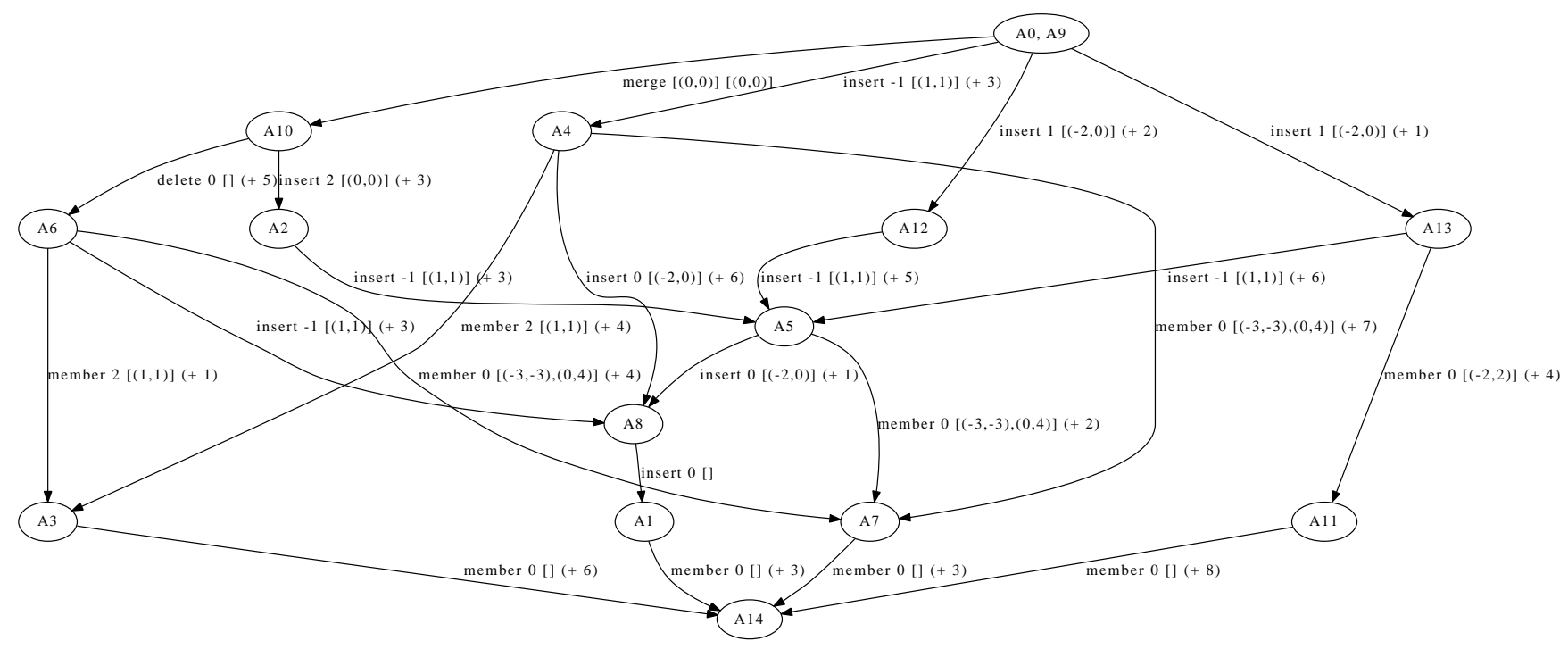

Figure 3: Relative correctness of interval set answers.

\begin{tabular}{l|c|c|c} 
Answer & Anon & IntSet & Crypto \\
\hline A0 & 16 & 16 & 4 \\
A1 & $0^{*}$ & $4^{*}$ & $0^{*}$ \\
A2 & $9^{*}$ & $11^{*}$ & 0 \\
A3 & 6 & $7^{*}$ & $0^{*}$ \\
A4 & $9^{*}$ & $12^{*}$ & $0^{*}$ \\
A5 & 10 & 7 & $4^{*}$ \\
A6 & 0 & 9 & $2^{*}$ \\
A7 & $12^{*}$ & 4 & $0^{*}$ \\
A8 & $16^{*}$ & $5^{*}$ & 0 \\
A9 & 7 & 16 & 2 \\
A10 & 0 & $15^{*}$ & 3 \\
A11 & 14 & 9 & $0^{*}$ \\
A12 & $10^{*}$ & 13 & 0 \\
A13 & $13^{*}$ & $14^{*}$ & 4
\end{tabular}

Figure 5: Results of automatic grading.

were not defined - and which therefore fail all tests. Interestingly, our analysis distinguishes all other answers - no two partially correct submissions were equivalent. Moreover, there is a non-trivial partial ordering of answers in each case: some answers really are strictly better than others. We conclude that our analysis is able to classify partially correct answers in an interesting way. (We also conclude that the cryptarithm problem was too hard to solve in the time available, since more than half of the submissions failed every test).

The final score assigned to each answer is shown in figure 5. In order to assign better answers a higher score, we show the number of tests passed by each answer, rather than the number of test failures - i.e. bugs. $A 0$ is the model answer in each case, and answers coming from the group assigned to using QuickCheck are marked with $\operatorname{stars}\left({ }^{*}\right)$.

The following table shows a statistical analysis of scores from the automatic ranking. To determine whether there is a statistical difference between samples coming from the two groups we applied Welch's $t$-test (which tests whether two collections of data have the same mean) and got the values visible in the $\mathrm{P}$-value row (which we shall explain below).

\begin{tabular}{l|c|c|c} 
& Anon & IntSet & Crypto \\
\hline All - Avg (Sdev) & $8.15(5.38)$ & $9.69(4.15)$ & $1.15(1.63)$ \\
QC - Avg (Sdev) & $9.86(5.01)$ & $9.71(4.39)$ & $0.86(1.57)$ \\
HU - Avg (Sdev) & $6.17(5.53)$ & $9.67(4.27)$ & $1.50(1.76)$ \\
P-value & 0.2390 & 0.9846 & 0.5065
\end{tabular}

For the anonymizer example, we can see that solutions developed using QuickCheck scored higher than those developed using HUnit, for interval sets the scores were about the same, and for the cryptarithm example, then solutions developed using QuickCheck fared worse. The P-value is the probability of seeing the observed (or lower) difference in scores by sheer chance, if there is no difference in the expected score using HUnit and QuickCheck (the null hypothesis). For the anonymizer problem then the null hypothesis can be rejected with a confidence of $76 \%$ - which is encouraging, but falls short of statistical significance (which would require a confidence of $95 \%$ or higher).

\subsection{Stability of the automatic bug measure}

Because our bug analysis does perform a random search in the space of test cases to construct its test suite, it is possible that we select a different set of tests, and thus assign a different rank to the same program in different runs. To investigate this, we ran the bug analysis ten times on the solutions to each of the three problems. We found that the partial ordering on solutions that we inferred did not change, but the size of test suite did vary slightly. This could lead to the same answer failing a different number of tests in different runs, and thus to a different rank being assigned to it. The table below shows the results for each problem. Firstly, the number of consecutive tests we ran without refining the test suite before concluding it was stable. Secondly, the sizes of the test suites we obtained for each problem. Once a test suite was obtained, we assigned a rank to each answer, namely the number of tests it failed. These ranks did differ between runs, but no answer was assigned ranks different by more than one in different runs. The last rows 
show the average and maximum standard deviations of the ranks assigned to each answer.

\begin{tabular}{l|c|c|c} 
& Anon & IntSet & Crypto \\
\hline Number of tests & 10000 & 10000 & 1000 \\
Sizes of test suite & 15,16 & 15,16 & 4 \\
Avg std dev of ranks & 0.08 & 0.06 & 0 \\
Max std dev of ranks & 0.14 & 0.14 & 0
\end{tabular}

We conclude that the rank assignment is not much affected by random choices made as we construct the test suite.

\subsection{Manual Grading of Solutions}

In the table below we present that average scores (and their standard deviations) from the manual grading for the three problems. These numbers are not conclusive from a statistical point of view. Thus, for the manual grading we can not reject the null hypothesis. Nevertheless, there is a tendency corresponding to the results of the automatic grading in section 4.1. For example, in the E-Mail anonymizer problem the solutions that use QuickCheck are graded higher than the solutions that use HUnit.

\begin{tabular}{c|c|c|c} 
& Anon & IntSet & Crypto \\
\hline All - Avg (Sdev) & $4.07(2.78)$ & $4.46(2.87)$ & $2.15(2.91)$ \\
QC - Avg (Sdev) & $4.86(2.67)$ & $4.43(2.88)$ & $1.86(3.23)$ \\
HU - Avg (Sdev) & $3.17(2.86)$ & $4.50(3.13)$ & $2.50(2.74)$
\end{tabular}

To further justify our method for automatic ranking of the solutions, we would like to see a correlation between the automatic scores and the manual scores. However, we can not expect them to be exactly the same since the automatic grading is in a sense less forgiving. (The automatic grading measure how well the program actually works, while the manual grading measure "how far from a correct program" the solution is.) If we look in more detail on the scores to the E-Mail anonymizer problem, presented in the table below, we can see that although the scores are not identical, they tend to rank the solutions in a very similar way. The most striking difference is for solution $A 7$, which is ranked 4 th by the automatic ranking and 10 th by the manual ranking. This is caused by the nature of the problem. The identity function (the function simply returning the input, $A 14)$ is actually a rather good approximation of the solution functionality-wise. $A 7$ is close to the identity function-it does almost nothing, getting a decent score from the automatic grading, but failing to impress a human marker.

\begin{tabular}{l|c|c|c|c} 
Answer & Auto & Manual & Auto rank & Manual rank \\
\hline A1 & 0 & 3 & 11 & 8 \\
A2 & 9 & 3 & 7 & 8 \\
A3 & 6 & 2 & 10 & 10 \\
A4 & 9 & 5 & 7 & 4 \\
A5 & 10 & 4 & 5 & 5 \\
A6 & 0 & 0 & 11 & 13 \\
A7 & 12 & 2 & 4 & 10 \\
A8 & 16 & 9 & 1 & 1 \\
A9 & 7 & 4 & 9 & 5 \\
A10 & 0 & 1 & 11 & 12 \\
A11 & 14 & 8 & 2 & 2 \\
A12 & 10 & 4 & 5 & 5 \\
A13 & 13 & 8 & 3 & 2
\end{tabular}

\subsection{Assessment of Students' Testing}

As described in section 2.7, we checked the quality of each student's test code both manually and automatically (by counting how many submissions each test suite could detect a bug in). Figure 6 shows the results.

\begin{tabular}{r|r|r|r|r|r|r|r} 
& \multicolumn{7}{|c}{ Student number } \\
\hline QuickCheck & $\mathbf{1}$ & $\mathbf{2}$ & $\mathbf{3}$ & $\mathbf{4}$ & $\mathbf{5}$ & $\mathbf{6}$ & $\mathbf{7}$ \\
\hline Manual grading & 0 & 0 & 0 & 3 & 9 & 9 & 12 \\
Automatic grading & 0 & 0 & 0 & 0 & 8 & 10 & 11
\end{tabular}

\begin{tabular}{r|r|r|r|r|r|r} 
& \multicolumn{6}{|c|}{ Student number } \\
\hline HUnit & $\mathbf{8}$ & $\mathbf{9}$ & $\mathbf{1 0}$ & $\mathbf{1 1}$ & $\mathbf{1 2}$ & $\mathbf{1 3}$ \\
\hline Manual grading & 3 & 12 & 6 & 3 & 6 & 9 \\
Automatic grading & 0 & 5 & 5 & 6 & 7 & 8
\end{tabular}

Figure 6: Manual vs automatic grading of test suite quality.

The manual scores may be biased since all the authors are QuickCheck afficionados, so we would like to use them only as a "sanity check" to make sure that the automatic scores are reasonable. We can see that, broadly speaking, the manual and automatic scores agree.

The biggest discrepancy is that student 9 got full marks according to our manual grading but only 5/11 according to the automatic grading. The main reason is that his test suite was less comprehensive than we thought: it included several interesting edge cases, such as an insert that "fills the gap" between two intervals and causes them to become one larger interval, but left out some simple cases, such as insert 2 (insert 0 empty). In this case, the automatic grader produced the fairer mark.

So, the automatically-produced scores look reasonable and we pay no more attention to the manual scores. Looking at the results, we see that four students from the QuickCheck group were not able to detect any bugs at all. (Three of them submitted no test code at all ${ }^{5}$, and one of them just tested one special case of the member function.) This compares to just one student from the HUnit group who was unable to find any bugs.

However, of the students who submitted a useful test suite, the worst QuickCheck test suite got the same score as the best HUnit test suite! All of the HUnit test suites, as it happens, were missing some edge case or other. ${ }^{6}$

So, of the students who were using QuickCheck, half failed to submit any useful test-suite at all, and the other half's test suites were the best ones submitted. There may be several explanations for this: perhaps QuickCheck properties are harder to write but more effective than unit tests; or perhaps QuickCheck is only effective in the hands of a strong programmer; or perhaps QuickCheck properties are "all-or-nothing", so that a property will either be ineffective or catch a wide range of bugs; or perhaps it was just a coincidence. This is something we will aim to find out in our next experiment.

\footnotetext{
${ }^{5}$ Of course, this does not imply that these students did not test their code at all - just that they did not automate their tests. Haskell provides a read-eval-print loop which makes interactive testing quite easy.

${ }^{6}$ Functions on interval sets have a surprising number of edge cases; with QuickCheck, there is no need to enumerate them.
} 


\section{RELATED WORK}

Much work has been devoted to finding representative test-suites that would be able to uncover all bugs even when exhaustive testing is not possible. When it is possible to divide the test space into partitions and assert that any fault in the program will cause one partition to fail completely it is enough select only a single test case from each partition to provoke all bugs. The approach was pioneered by Goodenough and Gerhart[?] who looked both at specifications and the control structure of tested programs and came up with test suites that would exercise all possible combinations of execution conditions. Weyuker and Ostrand[?] attempted to obtain good test-suites by looking at execution paths that they expect to appear in an implementation based on the specification. These methods use other information to construct test partitions, whereas our approach is to find the partitions by finding faults in random testing.

Lately, test-driven development has gained in popularity, and in a controlled experiment from 2005 [7] Erdogmus et. al. compare its effectiveness with a traditional test-after approach. The result was that the group using TDD wrote more test cases, and tended to be more productive. These results are inspiring, and the aim with our experiment was to show that property-based testing (using QuickCheck) is a good way of conducting tests in a development process.

In the design of the experiments we were guided by several texts on empirical research in software engineering, amongst which $[3,16,11]$ were the most helpful.

\section{CONCLUSIONS}

We have designed an experiment to compare propertybased testing and conventional unit testing, and as part of the design we have developed an unbiased way to assess the "bugginess" of submitted solutions. We have carried out the experiment on a small-scale, and verified that our assessment method can make fine distinctions between buggy solutions, and generates useful results. Our experiment was too small to yield a conclusive answer to the question it was designed to test. In one case, the interval sets, we observed that all the QuickCheck test suites (when they were written) were more effective at detecting errors than any of the HUnit test suites. Our automated analysis suggests, but does not prove, that in one of our examples, the code developed using QuickCheck was less buggy than code developed using HUnit. Finally, we observed that QuickCheck users are less likely to write test code than HUnit users - even in a study of automated testing - suggesting perhaps that HUnit is easier to use.

The main weakness of our experiment (apart from the small number of subjects) is that students did not have enough time to complete their answers to their own satisfaction. We saw this especially in the cryptarithm example, where more than half the students submitted solutions that passed no tests at all. In particular, students did not have time to complete a test suite to their own satisfaction. We imposed a hard deadline on students so that development time would not be a variable. In retrospect this was probably a mistake: next time we will allow students to submit when they feel ready, and measure development time as well.

In conclusion, our results are encouraging and suggest that a larger experiment could demonstrate interesting differences in power between the two approaches to testing. We look forward to holding such an experiment in the future.

\section{REFERENCES}

[1] T. Arts, J. Hughes, J. Johansson, and U. Wiger. Testing telecoms software with Quviq QuickCheck. In ERLANG '06: Proceedings of the 2006 ACM SIGPLAN workshop on Erlang, pages 2-10, New York, NY, USA, 2006. ACM.

[2] V. R. Basili and R. W. Selby. Comparing the effectiveness of software testing strategies. IEEE Trans. Softw. Eng., 13(12):1278-1296, 1987.

[3] V. R. Basili, R. W. Selby, and D. H. Hutchens. Experimentation in software engineering. IEEE Trans. Softw. Eng., 12(7):733-743, 1986.

[4] A. Bertolino. Software testing research: Achievements, challenges, dreams. In FOSE '07: 2007 Future of Software Engineering, pages 85-103, Washington, DC, USA, 2007. IEEE Computer Society.

[5] J. R. Brown and M. Lipow. Testing for software reliability. SIGPLAN Not., 10(6):518-527, 1975.

[6] K. Claessen and J. Hughes. QuickCheck: a lightweight tool for random testing of Haskell programs. In ICFP '00: Proc. of the fifth ACM SIGPLAN international conference on Functional programming, pages 268-279, New York, NY, USA, 2000. ACM.

[7] H. Erdogmus, M. Morisio, and M. Torchiano. On the effectiveness of the test-first approach to programming. IEEE Transactions on Software Engineering, 31:226-237, 2005.

[8] D. Herington. HUnit: A unit testing framework for haskell. http://hackage.haskell.org/package/HUnit-1.2.2.1, January 2010.

[9] JUnit.org. JUnit.org resources for test driven development. http://www.junit.org/, January 2010.

[10] S. King, J. Hammond, R. Chapman, and A. Pryor. Is proof more cost-effective than testing? IEEE Transactions on Software Engineering, 26:675-686, 2000.

[11] B. A. Kitchenham, S. L. Pfleeger, L. M. Pickard, P. W. Jones, D. C. Hoaglin, K. E. Emam, and J. Rosenberg. Preliminary guidelines for empirical research in software engineering. IEEE Transactions on Software Engineering, 28:721-734, 2002.

[12] G. J. Myers. A controlled experiment in program testing and code walkthroughs/inspections. Commun. ACM, 21(9):760-768, 1978.

[13] The GHC Team. The Glasgow Haskell Compiler. http://www.haskell.org/ghc/, January 2010.

[14] N. Tillmann and J. de Halleux. Pex-white box test generation for .NET. In Tests and Proofs, volume 4966 of Lecture Notes in Computer Science, pages 134-153. Springer Berlin / Heidelberg, 2008.

[15] N. Tillmann and W. Schulte. Parameterized unit tests. SIGSOFT Softw. Eng. Notes, 30(5):253-262, 2005.

[16] C. Wohlin, P. Runeson, M. Höst, M. C. Ohlsson, B. Regnell, and A. Wesslén. Experimentation in software engineering: an introduction. Kluwer Academic Publishers, Norwell, MA, USA, 2000.

[17] A. Zeller. Isolating cause-effect chains from computer programs. In SIGSOFT '02/FSE-10: Proceedings of the 10th ACM SIGSOFT symposium on Foundations of software engineering, pages 1-10, New York, NY, USA, 2002. ACM. 